\title{
The Impact of Near-infrared in Plastic Surgery
}

\author{
Yohei Tanaka $^{1,2,3}$, Yuichiro Tsunemi ${ }^{2}$, Makoto Kawashima ${ }^{2}$ and Hiroshi Nishida ${ }^{3}$ \\ ${ }^{1}$ Clinica Tanaka Plastic, Reconstructive Surgery and Anti-aging Center, Matsumoto, Nagano, Japan \\ ${ }^{2}$ Department of Dermatology, Tokyo Women's Medical University, Tokyo, Japan \\ ${ }^{3}$ Department of Applied Life Sciences, Niigata University of Pharmacy and Applied Life Sciences, \\ Niigata, Japan
}

Correspondence should be addressed to: Yohei Tanaka; info@clinicatanaka.jp

Received 22 January 2013; Accepted 21 March 2013; Published 23 June 2013

Academic Editor: Maureen Susan Thorniley

Copyright (c) 2013 Yohei Tanaka, Yuichiro Tsunemi, Makoto Kawashima and Hiroshi Nishida. Distributed under Creative Commons CC-BY 3.0

\begin{abstract}
Many studies regarding near-infrared, have used near-infrared resources without a water filter or a cooling system, and have proven its thermal effects. With these methods near-infrared energy is mainly absorbed in the superficial tissues and cannot be delivered sufficiently to deeper tissues. As solar near-infrared is filtered by atmospheric water, a water filter is essential in order to simulate solar near-infrared. Thus, these approaches could not sufficiently evaluate the effects of incident solar near-infrared that reaches the human tissue. We have clarified that the near-infrared that simulates solar near-infrared non-thermally affects subcutaneous tissues, including muscle. Importantly, the biological effects of near-infrared have both beneficial applications and deleterious effects. Near-infrared induces dermal heating thermally and non-thermally induces collagen and elastin stimulation, which results in skin tightening, and induces long-lasting vasodilation that may prevent vasospasm and may be beneficial for ischemic disorders and flap surgeries. Near-infrared also non-thermally relaxes and weakens dystonic or hypertrophic muscles to reduce wrinkles and myalgia. Its long-lasting induction of subcutaneous adipocytes may have an application in volume augmentation. However, continuous near-infrared exposure may induce photoaging and thinning of superficial muscles, which results in skin ptosis. Protection against near-infrared should be strongly considered, as over half of the solar energy is near-infrared. Although plastic surgeons are not familiar with the effects of near-infrared, its potential appears to be high and significant. This paper reviews the effects of near-infrared and introduces the new findings and applications of the biological effects of near-infrared in the field of plastic surgery.
\end{abstract}

Keywords: Near-infrared; non-thermal; biological effects; photoaging.

\section{Introduction}

Many studies have demonstrated the effects of sun and ultraviolet (UV) exposure on human tissue. However, the long-term effects of near-infrared (NIR) exposure on human skin and subcutaneous tissues have not been well 
investigated. Although various types of UV blocking materials, such as sunblock, sunglasses, films, and fibers are often used to prevent skin damage from UV exposure, and despite the world-wide use of these blocking materials, unwanted physiological effects such as rosacea, erythema ab igne, long-lasting vasodilation (Tanaka, 2009a; Tanaka, 2011a), long-lasting muscle thinning (Tanaka, 2010a, 2011b), and sagging and skin ptosis still occur (Tanaka, 2011c; Tanaka, 2012a, b). Both superficial muscle thinning and skin ptosis develop with age, and though face lift surgeries and skin tightening procedures are proven effective treatments, the mechanisms underlying their development remain unclear.

We previously reported that NIR from 1100 to $1800 \mathrm{~nm}$ together with a waterfilter that excludes wavelengths between 1400 and $1500 \mathrm{~nm}$ penetrates deep into human tissue and it is absorbed by water in the skin (Tanaka, 2009a,b; Tanaka, 2010b; Tanaka, 2011d), hemoglobin in dilated vessels (Tanaka, 2009a; Tanaka, 2011a), myoglobin in the superficial muscle (Tanaka, 2010a; Tanaka, 2011b), and bone cortical mass, and it is scattered by adipose cells (Tanaka, 2011e; Tanaka, 2012b).

Notably, in order to experimentally simulate solar NIR that reaches the skin (Tanaka, 2012a, b); a water filter is required, as solar NIR is filtered by atmospheric water (Anderson, 1981; Gates, 1966). NIR increases the surface temperature and induces thermal effects, so a contact cooling is needed to pursue the properties of NIR (Tanaka, 2011c; Tanaka, 2012a, b).

NIR is used to treat wound healing disorders (Danno, 2001; Horwitz, 1999; Schramm, 2003) and malignant tumors (Bäumler, 1999; Dees, 2002; Kelleher, 1999; Orenstein, 1999). NIR induces dermal heating thermally and nonthermally induces collagen and elastin stimulation, which results in skin tightening. NIR also induces long-lasting vasodilation that may prevent vasospasm and may be beneficial for ischemic disorders and flap surgeries in the field of plastic surgery. Further, NIR nonthermally relaxes and weakens dystonic or hypertrophic muscles to reduce wrinkles and myalgia.

In addition to its therapeutic effects, however, NIR also induces non-thermal DNA damage (Tanaka, 2010c; Tanaka, 2012c) and cell death by apoptosis (Tirlapur, 2001). NIR induces cell death of cancer cells and bone marrow cells (Tanaka, 2011e), which may have a potential application in the treatment of cancer (Tanaka, 2010c; Tanaka, 2012c).

The necessity to protect against NIR has not been well investigated to date. Fair skin with lower concentrations of melanin and a thin dermis might allow NIR to penetrate deeper into human tissue, and damage superficial muscles compared to darker skin with dense melanin and a thick dermis (Tanaka, 2011c; Tanaka, $2012 \mathrm{a}, \mathrm{b})$. In addition to natural NIR, human skin is increasingly exposed to artificial NIR from medical devices and electrical appliances (Schieke, 2003; Schroeder, 2008). Furthermore most sunscreens only block UV, but not visible light and NIR (Tanaka, 2011c; Tanaka, 2012a, b), and sunglasses and glasses are unable to block NIR exposure. Thus, individuals using sunscreens and glasses should further equip themselves for protection against NIR (Darvin, 2010; Meinke, 2011; Pujol, 1993; Schieke, 2003; Schroeder, 2008; Tanaka, 2011c; Tanaka, $2012 a$, b), as we are exposed to tremendous amounts of NIR (Tanaka, 2011c).

Previous studies regarding NIR have reported its application in the industrial and the agricultural fields, but have not well investigated the effects of NIR exposure in the medical fields, particularly in the field of plastic surgery.

Findings from our previous studies suggest that we should consider the biological effects of NIR, which have both potentially usefully applications and detrimental physiological effects. 


\section{Methods to Evaluate Non-Thermal Biological Effects of NIR}

In previous studies of NIR, a light source emitting wide wavelengths of NIR were used as an NIR source (Frank, 2004; Tanaka, 2012a). The temperature of the superficial layer of a culture fluid in a laboratory dish under NIR irradiation rises immediately, as NIR is mainly absorbed by water. The energy of NIR then decreases as it penetrates deeper, and will not reach enough target cells in the base. Therefore, these previous studies were only able to describe the optical and thermal effects of NIR, and are not useful to examine nonthermal biological effects of NIR (Tanaka, 2012a, b).

NIR induces dermal heating, which results in the tightening of skin laxity (Bitter, 2000; Chan, 2008; Goldberg, 2000; Ross, 2000; Tanaka, 2009a, b; Zelickson, 2006). In previous studies (Danno, 2001; Kim, 2005: Kligman, 1982) NIR devices without a water filter or contact cooling were used to investigate thermal effects on the human body. NIR increases the skin surface temperature and induces perspiration and vasodilation, as NIR is primarily absorbed by water and hemoglobin. Subsequently, a substantial amount of energy is absorbed in the superficial layers of skin, and only limited NIR energy can be delivered to deeper tissues. Therefore, these previous studies only describe superficial and thermal effects of NIR, and are not useful to examine non-thermal biological effects (Tanaka, 2012a, b).

Sunlight that reaches the human skin contains solar energy composed of $6.8 \%$ UV light, 38.9\% visible light and 54.3\% infrared (IR) (Kochevar, 1999). The IR spectral region is arbitrarily divided according to wavelength into sub-regions of NIR (760-3000 nm), middle IR (3000$30000 \mathrm{~nm}$ ), and far IR (30 $000 \mathrm{~nm}-1 \mathrm{~mm}$ ). NIR from the sun is selectively filtered by atmospheric water (Anderson, 1981; Gates, 1966), and NIR that reaches the Earth's surface readily penetrates the superficial layers of the skin (Tanaka, 2010a, 2011c, 2012a, 2011e) (Fig. 1, left).

Wavelengths below $1100 \mathrm{~nm}$ will be absorbed by melanin in the skin. Wavelengths between 1400 and $1500 \mathrm{~nm}$ and those above $1850 \mathrm{~nm}$ will be absorbed by water in the skin, which results in heating and may induce painful sensations and burns (Kelleher, 1999). Filtering out the wavelengths below 1100 $\mathrm{nm}$, around $1450 \mathrm{~nm}$, and above $1850 \mathrm{~nm}$ enabled the delivery of NIR to deeper tissues (Davenport, 2006) and also simulated the solar NIR that contracts human skin under natural conditions.

An NIR device that emits a spectrum of NIR wavelength from 1100 to $1800 \mathrm{~nm}$ together with a water-filter that excludes wavelengths between 1400 and $1500 \mathrm{~nm}$ as well as those above $1850 \mathrm{~nm}$ simulates the natural exposure of skin to NIR and allows for the evaluation of solar NIR that reaches the skin under natural conditions. NIR increases the surface temperature and induces thermal effects, thus, in order to reduce the skin surface temperature, perspiration, and blood vessel dilation, contact cooling is required. These specific wavelengths and the cooling system enables NIR to be delivered to the deeper tissues without pain or epidermal burns (Davenport, 2006; Goldberg, 2007), and allows for the investigations of properties of NIR (Tanaka, 2012a, b) (Fig. 1). 


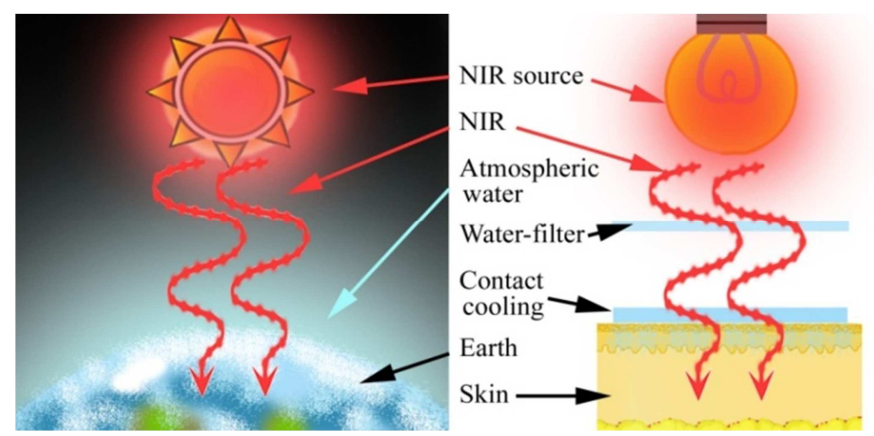

Fig. 1: A Schematic of Solar NIR (Left) and the NIR Research Strategy that Can Evaluate Non-Thermal Biological Effects of NIR (Right)

\section{Discussion}

\section{Properties and Chromophore of NIR}

NIR exhibits both wave and particle properties and it is strongly absorbed by water, hemoglobin, and myoglobin (Tanaka, 2011c). As a consequence, NIR can affect the subcutaneous tissues, including muscles, with its high permeability (Tanaka, 2011c) (Fig. 2).

The NIR spectrum is a result of the overtones and combination of bond stretching vibrations from $\mathrm{O}-\mathrm{H}, \mathrm{C}-\mathrm{H}$, and $\mathrm{N}-\mathrm{H}$ groups (Weyer, 1985). Water is a polar molecule possessing hydrogen bonds with an electrical dipole moment, and will be resonated by NIR. NIR will be resonated and absorbed in $\mathrm{O}-\mathrm{H}$ intramolecular hydrogen bonds and the electrical dipole moment (Tsai, 2001).

NIR induces an increase in water retention in the dermis and induces perspiration, vasodilation, and the expression of collagen and elastin (Tanaka, 2009a). Both collagen and elastin possess helical structures rich in hydrogen bonds. Humans have biological defense mechanisms in which induced hydrogen bonds and helical structures are resonated by NIR and absorb NIR to protect the subcutaneous tissues against this radiation.

NIR non-thermally induces degeneration of myoglobin, resulting in apoptosis of vascular smooth muscle cells and longlasting vasodilation (Tanaka, 2011a). Both hemoglobin and myoglobin have heme binding sites and alpha helices. Our results showing long-lasting muscle thinning and vasodilation induced by NIR suggest that NIR might resonate and damage heme. However, our collagen, elastin, and cancer studies suggest that NIR may mainly resonate hydrogen bonds, helical structures, alpha helices, and DNA (Tanaka, 2011c). Alpha helices are thought to be resonated by NIR (Nevskaya, 1976). Both hemoglobin and myoglobin are oxygen-carrying proteins with many alpha helices. It is possible that NIR induces resonance of helical structures in the oxygen-carrying proteins and degenerates proteins containing hydrogen bonds and helical structures, which result in damage to the storage and transport of oxygen. This could be one of the mechanisms of apoptosis (Tanaka, 2011c). 


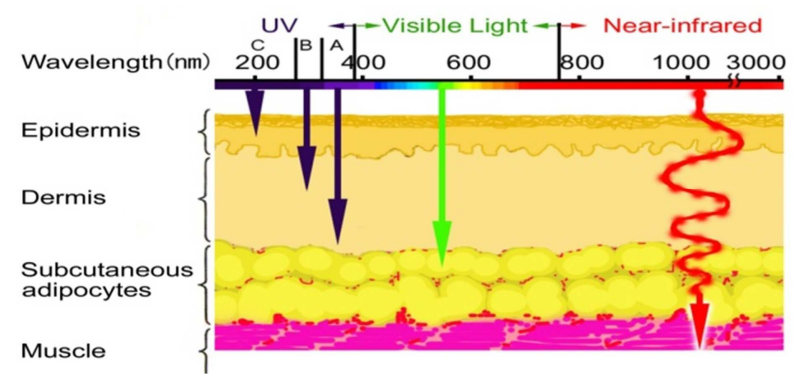

Fig. 2: A Schematic of Penetration Depth in Each Wavelength

\section{The Biological Effects of NIR}

NIR induces photochemical changes and affects a large volume and depth of tissue (Anderson, 1981). Actively proliferating cells show increased sensitivity to NIR (Karu, 1994; Tafur, 2008), and NIR induces strand breaks and apoptosis (Tirlapur, 2001) as well as death of cancer and bone marrow cells (Tanaka, 2010c, 2011e, 2012c). NIR is used to treat wound healing disorders (Danno, 2001; Horwitz, 1999; Schramm, 2003) and malignant tumors (Bäumler, 1999; Dees, 2002; Kelleher, 1999; Orenstein, 1999). While NIR appears to damage tumor tissue, it has also been shown to reduce cellular protein damage produced by biological oxidants in normal cells (Kujawa, 2004).

Subcutaneous adipocytes located above superficial muscles are effective and reasonable for the temperature retention and protection from NIR, because fatty tissue can scatter NIR optically (Tanaka, 2010a; Tanaka, 2011b) and fatty acids are the major NIR absorbing materials in soft tissues (Tsai, 2001). Oil in the liquid phase is transparent, whereas oil in the solid phase is highly scattering to NIR (Van Veen, 2005). The long-lasting induction of subcutaneous adipocytes may protect the underlying tissues, including the panniculus carnosus in animal and superficial muscles in human, against NIR damage. Because muscles contain hemoglobin and myoglobin which strongly absorb NIR, muscles are easily damaged by NIR (Srinivasan, 2003).

We previously reported that NIR thickens the dermis and induce the expression of collagen (Tanaka, 2009b), elastin, and water-binding proteins (Tanaka, 2009a, b) without scar formation (Tanaka, 2010b) to increase the amount of fluid protecting subcutaneous tissues from NIR. Further, NIR non-thermally induces vasodilation (Tanaka, 2011a), muscle thinning (Tanaka, 2010a), muscle relaxation (Tanaka, 2011b), bone marrow damage (Tanaka, 2011e), increased superficial granular adipocytes (Tanaka, 2011e), a cytocidal effect on cancer cells (Tanaka, 2010 c, 2012c), stimulation of stem cells (Tanaka, 2011c,e), and DNA damage of mitotic cells (Tanaka, 2010c, 2012c).

Other than the skin, the optic nerve which is the only exposed component of the central nervous system also has a defence mechanism against NIR on multiple levels; rich blood flow in the retina, hyaluronic acid in the lens, tears in sclera, and fat in the eyelid. These are effective protective mechanisms, as water, hemoglobin, and fat are all ideal materials for blocking exposure to and consequences of NIR.

Further studies are needed to investigate the range and mechanisms of the biological effects of NIR in humans.

\section{Beneficial Effects of NIR}

NIR induces dermal heating and nonthermally induces collagen and elastin stimulation, resulting in skin tightening (Tanaka, 2009a, b). NIR denatures collagen and promotes the generation of new collagen, resulting in tighter skin (Goldberg, 2007; Zelickson, 2006), and induces elevated levels of collagen production markers (Orringer, 2005). NIR also increases the amounts of collagen in human dermal fibroblasts, and can result 
in clinical improvement of skin texture (Lee, 2006). NIR induces high collagen density in the dermis, resulting in epidermal smoothness without scar formation, which provides safe, consistent, and long-term effects of skin rejuvenation (Tanaka, 2010b).

Pre-exposure of NIR prevents UV-induced toxicity (Danno, 1992; Frank, 2004; Menezes, 1998), and this effect is independent of heat shock protein induction and cell division (Menezes, 1998). These findings support the hypothesis that NIR prepares skin to better resist the subsequent damage from UV or NIR (Tanaka, 2012a, b).

NIR is known to be of therapeutic benefit in the treatment of musculoskeletal disorders and healing of indolent wounds (Ceylan, 2003; Webb, 1998). NIR induces longlasting vasodilation for an increase in blood circulation by causing apoptosis of vascular smooth muscle cells, which may prevent vasospasm and may be beneficial for ischemic disorders and flap surgeries.

NIR increases mitochondrial metabolism (Karu, 1999; Passarella, 1984; Yu, 1997; Wilden; 1998), facilitates wound healing, and promotes angiogenesis in skin (Conlan; 1996), bone (Yaakobi, 1996), nerve (Assia, 1989), and skeletal muscle (Bibikova, 1994; Oron, 2006).

NIR also non-thermally relaxes and weakens dystonic or hypertrophic muscles to reduce wrinkles and myalgia. Thus, this simple technique of using NIR may offer an alternative method to relax superficial muscles.

We previously reported that NIR increased subcutaneous adipocytes on the panniculus carnosus as well as CD34-positive cells surrounding the subcutaneous adipocytes. Adipogenesis is tightly associated with angiogenesis, and the expression of adipocytes is linked to the development of its vasculature (Christiaens, 2010). Thus, induction of subcutaneous adipocytes may have an application for volume augmentation.

Our previous study revealed that NIR increased subcutaneous and bone marrow adipocytes, CD34-positive hematopoietic stem cells in bone marrow, and cortical bone mass (Tanaka, 2011e). Stimulation of stem cells by NIR irradiation might be beneficial for applications in regenerative medicine.

NIR is also an essential tool in cancer detection and imaging, as actively proliferating cells show increased sensitivity to NIR (Karu, 1994; Tafur, 2008). NIR also induces non-thermal DNA damage of mitotic cells especially in prophase, metaphase, and anaphase due to the absence of nuclear lamin protection (Tanaka, 2010c, 2012c), and suppresses the proliferation of various kinds of malignant cells (Tanaka, 2010c, 2011c, 2012c).

NIR may be beneficial in multiple fields of plastic surgery, as the schedule reduces discomfort and side effects, deep subcutaneous tissues are accessible, and facilitates repeated irradiations.

These findings indicate that NIR has a wide range of biological effects, and future studies are warranted to develop these findings into potential useful and beneficial techniques and applications. 


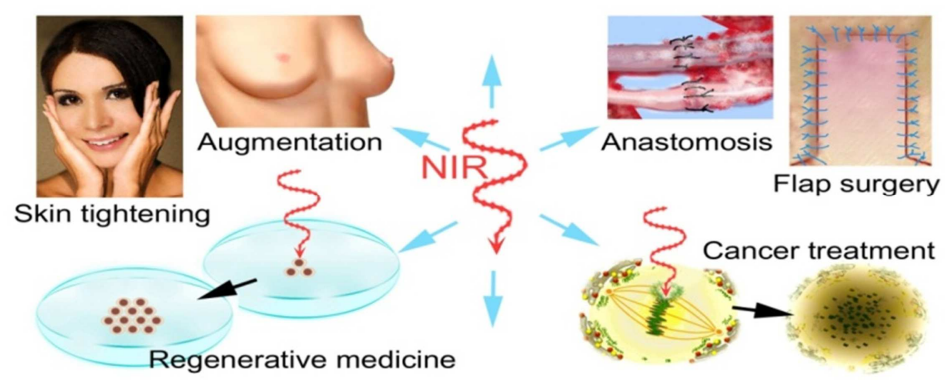

Fig. 3: Schematic Indicating Effects and Potentially Useful Applications of NIR

\section{Deleterious Effects of NIR}

NIR exerts biologic effects on human skin (Schieke, 2003). NIR causes skin changes similar to those observed in solar elastosis, and enhance UV-induced dermal damage (Kligman, 1982). NIR activates mitogenactivated protein kinases and induces gene transcription, and it is likely to increase collagen degradation (Kim, 2006; Schieke, 2003; Schroeder, 2008). Epidemiological data and clinical reports imply that NIR is not innocuous to human skin (Dover, 1989; Kligman, 1984; Schieke, 2003).

Several diseases such as rosacea and erythema $a b$ igne might be induced by chronic NIR exposure. Rosacea is a chronic cutaneous disorder characterized by centrofacial persisting erythema, telangiectasia, papules, pustules, edema, and ocular involvement. Rosacea affects all races, though is more common in Caucasians and fair-skinned populations (Berg, 1989). NIR should be considered as a critical factor in the development and aggravation of rosacea, since the distribution of erythema is most prominent on the facial convexities (Bae, 2009).

Long-term exposure to sources of heat and NIR, such as fires and stoves, results in erythema ab igne (Findlayson, 1966), which is clinically characterized by a reticular hyperpigmentation and telangiectasia accompanied histologically by epidermal atrophy, vasodilation, dermal melanin and hemosiderin deposits. Longterm exposure of NIR from various heat sources is thought to induce reticulated erythema and results in histopathological changes similar to those seen in solardamaged skin (Page, 1988). The occurrence of telangiectasia appeared to increase with age, increased sunbathing, and poor pigmentation ability (Berg, 1989).

These lesions may develop thermal keratosis, such as hyperkeratosis, keratinocyte dysplasia, and dermal elastosis, which are similar to the changes that occur in actinically damaged skin (Arrington, 1979). Several reports indicate that carcinomas arise from heatinduced erythema ab igne (Hewitt, 1993; Jones, 1988; Kligman, 1984). Similar to UV, NIR induces photoaging and potentially photocarcinogenesis (Schieke, 2003). In addition, skin tumors in mice appeared faster after irradiation with the full lamp spectrum containing UV, visible, and NIR compared to irradiation with UV alone (Bain, 1943).

NIR is attenuated by thick watercontaining dermis. Thus, skin with sparse melanin and a thin dermis allows NIR to penetrate deeper into tissue than skin with dense melanin and a thick dermis (Tanaka, 2011c; Tanaka, 2012a, b). The mean area of the facial surface that is covered with wrinkles is significantly larger in Caucasians than in African Americans, and characteristics of agerelated periorbital changes seem to occur at a more accelerated rate in Caucasians (Odunze, 2008). In addition, fair skin is more sensitive to skin aging (Guinot, 2002; Nagashima, 1999). These findings support the observation that fair skin tends to wrinkle and sag earlier in life (Rawlings, 2006; Tsukahara, 2004), as fair skin is thinner and is more susceptible to NIR damage to the underlying superficial muscles than dark skin (Tanaka, 2011c; Tanaka, 2012a). 
Continual long-term exposure to solar NIR causes superficial muscle thinning and the muscle extensions to the dermis, which ultimately leads to skin ptosis (Fig. 4). Additional factors thought to contribute to brow ptosis include the gradual loss of forehead skin elasticity and a reduction in the tone of the frontalis muscles (Knize, 1996; Niechajev, 2004). The use of NIR for smoothing forehead wrinkles also caused brow ptosis. Although further studies are required to confirm our results, these results may have major implications in superficial tissue aging and skin ptosis.
Plastic and reconstructive surgeons are usually exposed to NIR from the microscope for an extended period of time during microsurgery, thus, surgeons should consider protection of eyes from NIR. NIR that is absorbed by the anterior segment (the cornea, aqueous, and lens) can produce clouding of the cornea and cataract (Aly, 2011; Lydahl, 1984; Zaret, 1976). The International Commission on Non-Ionizing Radiation Protection recommended protection to avoid the thermal injury of the cornea and possible cataractogenesis.

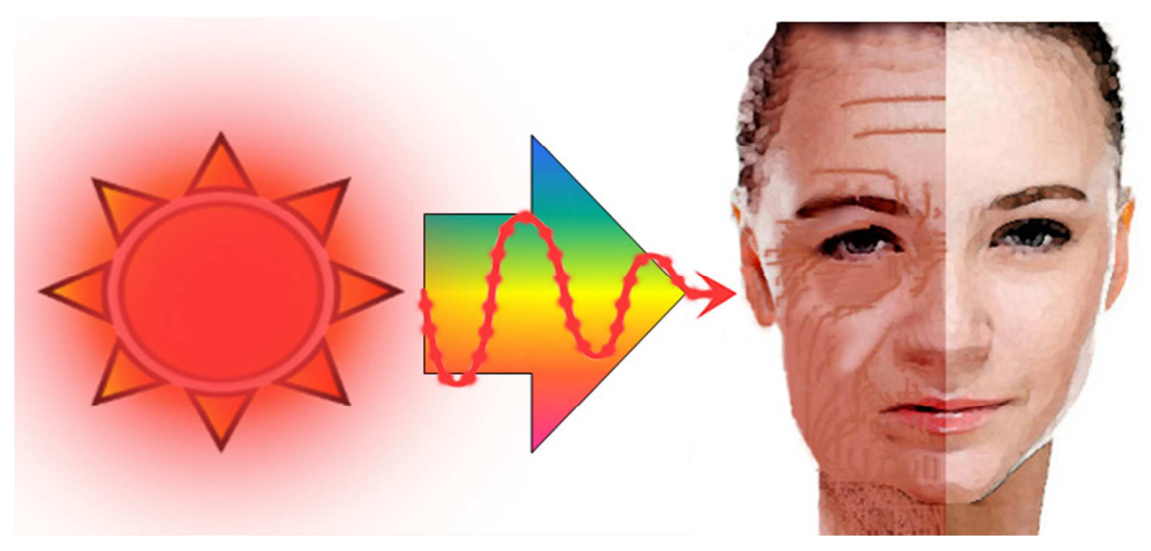

Fig. 4: Superficial Tissue Aging Induced by Solar NIR Radiation

\section{Conclusion}

In order to simulate solar NIR that reaches the skin and to investigate the properties of NIR, the NIR source must possess a water filter and contact cooling.

Appropriate NIR induces dermal heating thermally and non-thermally induces collagen and elastin stimulation, which results in tightening of skin laxity. NIR also non-thermally induces long-lasting vasodilation, which may prevent vasospasm and may be beneficial for ischemic disorders, vasospasm, and flap surgeries. Further, NIR relaxes and weakens dystonic or hypertrophic muscles to reduce wrinkles and myalgia, which might have an application for treating muscle disorders, and causes long-lasting induction of subcutaneous adipocytes, which may have an application for volume augmentation. NIR might be beneficial for regenerative medicine based on its stimulation of stem cells. Cancer detection and imaging may gain from NIR, due to cancer's high sensitivity to NIR, and NIR induction of non-thermal DNA damage of mitotic cells could be useful in cancer treatment. These new techniques using NIR may be beneficial in many fields of plastic surgery.

Importantly, solar NIR may cause unexpected photoaging, muscle thinning and stimulation of stem cells, including cancer stem cells, in areas of the body exposed to the sun. Although various kinds of sunscreen materials are often used to prevent skin damage from UV exposure, these materials block neither visible light nor NIR. Therefore, skin should be protected with clothing or sunscreen that not only blocks UV, but also NIR, in order to prevent photoaging, skin ptosis, and oncogenicity. However, additional non- 
thermal studies are needed to investigate the biological effects of NIR in humans.

\section{Disclosure}

None of the authors of this study have a conflict of interest.

\section{References}

Aly, E. M. \& Mohamed, E. S. (2011). "Effect of Infrared Radiation on the Lens," Indian journal of ophthalmology, 59 97-101.

Anderson, R. R. \& Parrish, J. A. (1981). "The Optics of Human Skin," Journal of Investigative Dermatology, 77 13-19.

Arrington, J. H. III. \& Lockman, D. S. (1979). "Thermal Keratoses and Squamous Cell Carcinoma in Situ Associated with Erythema Ab Igne," Archives of Dermatology, 115 1226-1228.

Assia, E., Rosner, M., Belkin, M., Solomon, A. \& Schwartz, M. (1989). "Temporal Parameters of Lowenergy Laser Irradiation for Optimal Delay of Post-Traumatic Degeneration of Rat Optic Nerve," Brain Research, 476 205-212.

Bae, Y. I., Yun, S. J., Lee, J. B., Kim, S. J., Won, Y. H. \& Lee, S. C. (2009). "Clinical Evaluation of 168 Korean Patients with Rosacea: The Sun Exposure Correlates with the Erythematotelangiectatic Subtype," Annals of Dermatology, 21 (3) 243-249.

Bain, J. A, Rusch, H. P. \& Kline, B. E. (1943). "The Effect of Temperature upon Ultraviolet Carcinogenesis with Wavelength 2,800-3,400A ${ }^{\circ}$,"Cancer Research, 3 610-612.

Bäumler, W., Abels, C., Karrer, S., Weiss, T., Messmann, H., Landthaler, M. \& Szeimies, R.- M. (1999). "Photo-Oxidative Killing of Human Colonic Cancer Cells Using Indocyanine Green and Infrared Light," British Journal of Cancer, 80 (3-4) 360-363.

Berg, M. (1989). "Epidemiological Studies of the Influence of Sunlight on the Skin," Photodermatol, 6 80-84.
Bibikova, A. \& Oron, U. (1994). "Attenuation of the Process of Muscle Regeneration in the Toad Gastrocnemius Muscle by Low Energy Laser Irradiation," Lasers in Surgery and Medicine, 14 355-361.

Bitter, P. H. (2000). "Noninvasive Rejuvenation of Photodamaged Skin Using Serial, Full-Face Intense Pulsed Light Treatments," Dermatologic Surgery, 26 835-843.

Ceylan, Y., Hizmetli, S. \& Siling, Y. (2003). "The Effects of Infrared Laser and Medical Treatments on Pain and Serotonin Degradation Products in Patients with Myofascial Pain Syndrome: A Controlled Trial," Rheumatology International, 24 260263.

Chan, H. H., Yu, C. S., Shek, S., Yeung, C. K., Kono, T. \& Wei, W. I. (2008). "A Prospective, Split Face, Single-Blinded Study Looking at the Use of an Infrared Device with Contact Cooling in the Treatment of Skin Laxity in Asians," Lasers in Surgery and Medicine, 40 146-152.

Christiaens, V. \& Lijnen, H. R. (2010). "Angiogenesis and Development of Adipose Tissue. Review," Molecular and Cellular Endocrinology, 318 2-9.

Conlan, M. J., Rapley, J. W. \& Cobb, C. M. (1996). "Biostimulation of Wound Healing by Low-Energy Laser Irradiation," Journal of Clinical Periodontology, 23 492-496.

Danno, K., Horio, T. \& Imamura, S. (1992). "Infrared Radiation Suppresses Ultraviolet B-Induced Sunburn-Cell Formation," Archives of Dermatological Research, 284 92-94.

Danno, K., Mori, N., Toda, K., Kobayashi, T. \& Utani, A. (2001). "Near-Infrared Irradiation Stimulates Cutaneous Wound Repair: Laboratory Experiments on Possible Mechanisms," Photodermatology, Photoimmunology \& Photomedicine, 17 261-265. 
Darvin, M. E., Haag, S., Meinke, M., Zastrow, L., Sterry, W. \& Lademann, J. (2010). "Radical Production by Infrared a Irradiation in Human Tissue," Skin Pharmacol Physiol, 23 (1) 40-46.

Davenport, S. A., Gollnick, D. A., Levernier, M. \& Spooner, G. J. R. (2006). "Method and System for Treatment of Post-Partum Abdominal Skin Redundancy or Laxity," United States Patent 20060052847. Available at: http://www.freepatentsonline.com/y2006 /0052847.html

Dees, C., Harkins, J., Petersen, M. G., Fisher, W. G. \& Wachter, E. A. (2002). "Treatment of Murine Cutaneous Melanoma with Near Infrared Light," Photochemistry and Photobiology, 75 296-301.

Dover, J. S., Phillips, T. J. \& Arndt, K. A. (1989). "Cutaneous Effects and Therapeutic Uses of Heat with Emphasis on Infrared Radiation," Journal of the American Academy of Dermatology, 20 278-286.

Findlayson, G. R., Sams, W. M. Jr. \& Smith, J. G. (1966). "Erythema Ab Igne: A Histopathological Study," The Journal of Investigative Dermatology, 46 104-107.

Frank, S., Oliver, L., Lebreton-De, Coster, C., Moreau, C., Lecabellec, M. T., Michel, L., Vallette, F. M., Dubertret, L. \& Coulomb, B. (2004). "Infrared Radiation Affects the Mitochondrial Pathway of Apoptosis in Human Fibroblasts," Journal of Investigative Dermatology, 123 823-831.

Gates, D. M. (1966). "Spectral Distribution of Solar Radiation at the Earth's Surface," Science, 151 523-529.

Goldberg, D. J. (2000). "New Collagen Formation after Dermal Remodeling with an Intense Pulsed Light Source," Journal of Cosmetic and Laser Therapy, 2 59-61.

Goldberg, D. J., Hussain, M., Fazeli, A. \& Berlin, A. L. (2007). "Treatment of Skin Laxity of the Lower Face and Neck in Older Individuals with a Broad Spectrum Infrared Light Device," Journal of Cosmetic and Laser Therapy, 9 35-40.
Guinot, C., Malvy, D. J.- M., Ambroisine, L., Latreille, J., Mauger, E., Tenenhaus, M., Morizot, F., Lopez, S., Le Fur, I. \& Tschachler, E. (2002). "Relative Contribution of Intrinsic Vs. Extrinsic Factors to Skin Aging as Determined by a Validated Skin Age Score," Arch Dermatol, 138 1454-1460.

Hewitt, J. B., Sherif, A., Kerr, K. M. \& Stankler, L. (1993). "Merkel Cell and Squamous-Cell Carcinomas Arising in Erythema Ab Igne," British Journal of Dermatology, 128 591-592.

Horwitz, L. R., Burke, T. J. \& Carnegie, D. (1999). "Augmentation of Wound Healing Using Monochromatic Infrared Energy. Exploration of a New Technology for Wound Management," Advances Wound Care, 12 (1) 35-40.

Jones, C. S., Tyring, S. K., Lee, P. C. \& Fine, J. D. (1988). "Development of Neuroendocrine (Merkel Cell) Carcinoma Mixed with Squamous-Cell Carcinoma in Erythema Ab Igne," Archives of Dermatology, 124 110-113.

Karu, T. (1999). "Invited Review. Primary and Secondary Mechanisms of Action of Visible to Near-IR Radiation on Cells," Journal of Photochemistry and photobiology B: Biology, 49 1-17.

Karu, T., Pyatibrat, L. \& Kalendo, G. (1994). "Irradiation with $\mathrm{He}-\mathrm{Ne}$ Laser Can Influence the Cytotoxic Response of Hela Cells to Ionizing Radiation," International Journal of Radiation Biology, 65 691-697.

Kelleher, D. K., Thews, O., Rzeznik, J., Scherz, A., Salomon, Y. \& Vaupel, P. (1999). "Hot Topic. Water-Filtered Infrared-A Radiation: A Novel Technique for Localized Hyperthermia in Combination with Bacteriochlorophyll-Based Photodynamic Therapy," International Journal of Hyperthermia, 15 467-474.

Kim, H. H., Lee, M. J., Lee, S. R., Kim, K. H., Cho, K. H., Eun, H. C. \& Chung, J. H. (2005). "Augmentation of UV-Induced Skin Wrinkling by Infrared Irradiation in 
Hairless Mice," Mechanisms of Ageing and Development,126 1170-1177.

Kim, M. S., Kim, Y. K., Cho, K. H. \& Chung, J. H. (2006). "Infrared Exposure Induces an Angiogenic Switch in Human Skin That is Partially Mediated by Heat," British Journal of Dermatology, 155 1131-1138.

Kligman, L. H. (1982). "Intensification of Ultraviolet-Induced Dermal Damage by Infrared Radiation," Archives of Dermatological Research, 272 229-238.

Kligman, L. H. \& Kligman, A. M. (1984). "Reflections on Heat," British Journal of Dermatology, 110 369-375.

Knize, D. M. (1996). "An Anatomically Based Study of the Mechanism of Eyebrow Ptosis," Plastic and Reconstructive Surgery, 97 1321-1333.

Kochevar, I. E., Pathak, M. A. \& Parrish, J. A. (1999). 'Photophysics, Photochemistry and Photobiology,' In: Freedberg, I. M, Eisen, A. Z, Wolff, K, et al, Eds. Fitzpatrick's Dermatology in General Medicine. New York: Mcgraw-Hill: 220-229.

Kujawa, J., Zavodnik, I. B., Lapshina, A., Labieniec, M. \& Bryszewska, M. (2004). "Cell Survival, DNA, and Protein Damage in B14 Cells under Low-Intensity NearInfrared (810nm) Laser Irradiation," Photomedicine and Laser Surgery, 22 504508.

Lee, J. H., Roh, M. R. \& Lee, K. H. (2006). "Effects of Infrared Radiation on Skin Photo-Aging and Pigmentation," Yonsei Medical Journal, 47 (4) 485-490.

Lydahl, E. (1984). "Infrared Radiation and Cataract," Acta ophthalmologica. Supplementum, 166 1-63.

Meinke, M. C., Haag, S. F., Schanzer, S., Groth, N., Gersonde, I. \& Lademann, J. (2011). "Radical Protection by Sunscreens in the Infrared Spectral Range," Photochemistry and Photobiology, 87 (2) 452-456.
Menezes, S., Coulomb, B., Lebreton, C. \& Dubertret, L. (1998). "Non-Coherent Near Infrared Radiation Protects Normal Human Dermal Fibroblasts from Solar Ultraviolet Toxicity," Journal of Investigative Dermatology, 111 629-633.

Nagashima, H., Hanada, K. \& Hashimoto, I. (1999). "Correlation of Skin Phototype with Facial Wrinkle Formation," Photodermatology, Photoimmunology \& Photomedicine, 15 2-6.

Nevskaya, N. A. \& Chirgadze, Y. N. (1976). "Infrared Spectra and Resonance Interactions of Amide-I and II Vibrations of Alpha-Helix," Biopolymers, 15 (4) 637-648.

Niechajev, I. (2004). "Transpalpebral Browpexy," Plastic and Reconstructive Surgery, 113 2172-2180.

Odunze, M., Rosenberg, D. S. \& Few, J. W. (2008). "Periorbital Aging and Ethnic Considerations: A Focus on Leteral Canthal Complex," Plastic and Reconstructive Surgery, 121 1002-1008.

Orenstein, A., Kostenich, G., Kopolovic, Y., Babushkina, T. \& Malik, Z. (1999). "Enhancement of ALA-PDT Damage by IRInduced Hyperthermia on a Colon Carcinoma Model," Photochemistry and Photobiology, 69 (6) 703-707.

Oron, U. (2006). "Photoengineering of Tissue Repair in Skeletal and Cardiac Muscles," Photomedicine and Laser Therapy, 24 111-120.

Orringer, J. S., Voorhees, J. J., Hamilton, T., Hammerberg, C., Kang, S., Johnson, T. M., Karimipour, D. J. \& Fisher, G. (2005). "Dermal Matrix Remodeling after Nonablative Laser Therapy," Journal of the American Academy of Dermatology, 53 775782.

Page, E. H. \& Shear, N. H. (1988). "Temperature-Dependent Skin Disorders," Journal of the American Academy of Dermatology, 18 1003-1019. 
Passarella, S., Casamassima, E., Molinari, S., Pastore, D., Quagliariello, E., Catalano, I. M. \& Cingolani, A. (1984). "Increase of Proton Electrochemical Potential and ATP Synthesis in Rat Liver Mitochondria Irradiated in Vitro by Helium-Neon Laser," FEBS Letters,175 95-99.

Pujol, J. A. \& Lecha, M. (1993). "Photoprotection in the Infrared Radiation Range," Photodermatology, Photoimmunology \& Photomedicine, 9 275278.

Rawlings, A. V. (2006). "Ethnic Skin Types: Are There Differences in Skin Structure and Function? Review Article, " International Journal of Cosmetic Science, 28 79-93.

Ross, E. V., Sajben, F. P., Hsia, J., et al. (2000). "Non-Ablative Skin Remodeling: Selective Dermal Heating with a MidInfrared Laser and Contact Cooling Combination," Lasers in Surgery and Medicine, 26 186-195.

Schieke, S. M., Schroeder, P. \& Krutmann, J. (2003). "Review Article. Cutaneous Effects of Infrared Radiation: From Clinical Observations to Molecular Response Mechanisms," Photodermatology, Photoimmunology \& Photomedicine, 19 228-234.

Schramm, J. M., Warner, D., Hardesty, R. A. \& Oberg, K. C. (2003). "A Unique Combination of Infrared and Microwave Radiation Accelerates Wound Healing," Plastic \& Reconstructive Surgery, 111 (1) 258-266.

Schroeder, P., Lademann, J., Darvin, M. E., Stege, H., Marks, C., Bruhnke, S. \& Krutmann, J. (2008). "Infared RadiationInduced Matrix Metalloproteinase in Human Skin: Implications for Protection," Journal of Investigative Dermatology, 128 2491-2497.

Srinivasan, S., Pogue, B. W., Jiang, S., Dehghani, H., Kogel, C., Soho, S., Gibson, J. J., Tosteson, T. D., Poplack, S. P. \& Paulsen, D. K. (2003). "Interpreting Hemoglobin and Water Concentration, Oxygen Saturation, and Scattering Measured in Vivo by Near-
Infrared Breast Tomography," Proceedings of the National Academy of Sciences of the United States of America, 100 12349-12354.

Tafur, J. \& Mills, P. J. (2008). "Low-Intensity Light Therapy: Exploring the Role of Redox Mechanisms," Photomedicine and Laser Surgery, 26 321-326.

Tanaka, Y. (2012b). "Impact of NearInfrared in Dermatology, Review," World Journal of Dermatology, 1(3) 30-37.

Tanaka, Y. \& Kawashima, M. (2012a). 'The Biological Effects of Near-Infrared,' Aesthet Dermatol, 22, 100-109.

Tanaka, Y. \& Matsuo, K. (2011c). "NonThermal Effects of Near-Infrared Irradiation on Melanoma," Breakthroughs in Melanoma Research (Ed. Tanaka, Y.), ISBN: 978-953-307-291-3, Intech, 597-628.

Tanaka, Y., Matsuo, K. \& Yuzuriha, S. (2009a). "Long-Term Evaluation of Collagen and Elastin Following Infrared (1100 To $1800 \mathrm{Nm}$ ) Irradiation," Journal of Drugs in Dermatology, 8 708-712.

Tanaka, Y., Matsuo, K. \& Yuzuriha, S. (2010a). "Long-Lasting Muscle Thinning Induced by Infrared Irradiation Specialized with Wavelength and Contact Cooling: A Preliminary Report," Eplasty, 10:E40.

Tanaka, Y., Matsuo, K. \& Yuzuriha, S. (2010b). "Long-Term Histological Comparison between Near-Infrared Irradiated Skin and Scar Tissues," Clin Cosmet Investig Dermatol, 3 143-149.

Tanaka, Y., Matsuo, K. \& Yuzuriha, S. (2011a). "Near-Infrared Irradiation NonThermally Induces Long-Lasting Vasodilation by Causing Apoptosis of Vascular Smooth Muscle Cells," Eplasty, 11:E22.

Tanaka, Y., Matsuo, K. \& Yuzuriha, S. (2011b). "Long-Lasting Relaxation of Corrugator Supercilii Muscle Contraction Induced by Near Infrared Irradiation," Eplasty, 11:E6. 
Tanaka, Y., Matsuo, K. \& Yuzuriha, S. (2011d). "Objective Assessment of Skin Rejuvenation Using Near-Infrared 1064Nm Neodymium: YAG Laser in Asians," Clinical, Cosmetic and Investigational Dermatology, 4 123-130.

Tanaka, Y., Matsuo, K. \& Yuzuriha, S. (2011e). "Near-Infrared Irradiation NonThermally Affects Subcutaneous Adipocytes and Bone," Eplasty, 11:E12.

Tanaka, Y., Matsuo, K., Yuzuriha, S. \& Shinohara, H. (2009b). "Differential LongTerm Stimulation of Type I versus Type III Collagen after Infrared Irradiation," Dermatologic Surgery, 35 1099-1104.

Tanaka, Y., Matsuo, K., Yuzuriha, S., Yan, H. \& Nakayama, J. (2010c). "Non-Thermal Cytocidal Effect of Infrared Irradiation on Cultured Cancer Cells Using Specialized Device," Cancer Science, 101 1396-1402.

Tanaka, Y., Tatewaki, N., Nishida, H., Eitsuka, T., Ikekawa, N. \& Nakayama, J. (2012c). "Non-Thermal DNA Damage of Cancer Cells Using Near-Infrared Irradiation," Cancer Science, 103 14671473.

Tirlapur, U. K. \& König, K. (2001). "Femtosecond Near-Infrared Laser Pulse Induced Strand Breaks in Mammalian Cells," Cellular and Molecular Biology, 47 131-134.

Tsai, C.- L., Chen, J.- C. \& Wang, W.- J. (2001). "Near-Infrared Absorption Property of Biological Soft Tissue Constituents," Journal of Medical and Biological Engineering, 21 7-14.

Tsukahara, T., Fujimura, T., Yoshida, Y., Kitahara, T., Hotta, M., Moriwaki, S., Witt, P. S., Simion, F. A. \& Takema, Y. (2004). "Comparison of Age-Related Changes in Wrinkling and Sagging of the Skin in Caucasian Females and in Japanese Females," International Journal of Cosmetic Science, 55 373-385.
Van Veen, R. L., Sterenborg, H. J., Pifferi, A., Torricelli, A., Chikoidze, E. \& Cubeddu, R. (2005). "Determination of Visible Near-IR Absorption Coefficients of Mammalian Fat Using Time- and Spatially Resolved Diffuse Reflectance and Transmission Spectroscopy," Journal of Biomedical Optics, 10054004.

Webb, C., Dyson, M. \& Lewis, W. H. (1998). "Stimulatory Effect of 660-Nm Low Level Laser Energy on Hypertrophic Scarderived Fibroblasts: Possible Mechanisms for Increase in Cell Counts," Lasers in Surgery and Medicine, 22 294-301.

Weyer, L. G. (1985). "Near-Infrared Spectroscopy of Organic Substances," Applied Spectroscopy Reviews, 21 1-43.

Wilden, L. \& Karthein, R. (1998). "Import of Radiation Phenomena of Electrons and Therapeutic Low-Level Laser in Regard to the Mitochondrial Energy Transfer," Journal of Clinical Laser Medicine \& Surgery, $16159-165$.

Yaakobi, T., Maltz, L. \& Oron, U. (1996). "Promotion of Bone Repair in the Cortical Bone of the Tibia in Rats by Low Energy Laser (He-Ne) Irradiation," Calcified Tissue International, 59 297-300.

Yu, W., Naim, J. O., Mcgowan, M., Ippolito, K. \& Lanzafame, R. J. (1997). "Photomodulation of Oxidative Metabolism and Electron Chain Enzymes in Rat Liver Mitochondria," Photochemistry and Photobiology, 66 866-871.

Zaret, M. M., Snyder, W. Z. \& Birenbaum, L. (1976). "Cataract after Exposure to NonIonizing Radiant Energy," British Journal of Ophthalmology, 60 632-637.

Zelickson, B., Ross, V., Kist, D., Counters, J., Davenport, S. \& Spooner, G. (2006). "Ultrastructural Effects of an Infrared Handpiece on Forehead and Abdominal Skin," Dermatologic Surgery, 32 897-901. 